\title{
Quantum entanglement of nontrivial spacetime topology
}

\author{
Zhiming Huang ${ }^{\mathrm{a}}$ \\ School of Economics and Management, Wuyi University, Jiangmen 529020, China
}

Received: 16 December 2019 / Accepted: 4 February 2020 / Published online: 15 February 2020

(C) The Author(s) 2020

\begin{abstract}
We analyze the entanglement behaviors for two accelerating atoms interacting with a massless scalar field in the cosmic string spacetime. We calculate different correlation functions for different spacetime topologies. We find that entanglement behaviors are determined by the vacuum fluctuation, two-atom distance, acceleration and nontrivial spacetime topology. It is shown that great two-atom distance and acceleration have negative effect on quantum entanglement. The existence of string has profound impact on the atom-field interaction system and the entanglement behaviors. When deficit angle parameter $v=1$ and atoms are far away from the string, the entanglement behaviors are the same with that of Minkowski spacetime. Our analysis about entanglement behaviors in cosmic string spacetime in principle is beneficial to sense the cosmic string spacetime topology structure and property, and discriminate the cosmic string spacetime and Minkowski spacetime. Besides, we also discuss the Unruh thermal effect in the cosmic string spacetime.
\end{abstract}

\section{Introduction}

Quantum entanglement is identified as the most nonclassical feature of quantum formalism, which plays crucial role in quantum information science $[1,2]$. However, the inevitable coupling of quantum system with environment may destroy quantum entanglement, such as a quantum system coupling to the fluctuating quantum field. Recently, the entanglement dynamics is investigated for uniformly accelerated atoms coupling to fluctuating scalar field or electromagnetic field in Minkowski spacetime, and the results show that entanglement exhibit behaviors of degradation, generation, revival and enhancement under the influence of vacuum fluctuation [3,4]. Lately investigation of entanglement dynamics in curved spacetimes was implemented [5,6], and

\footnotetext{
a e-mail: 465609785@qq.com (corresponding author)
}

the research shows that entanglement behaviors can be utilized to tell the difference between the de Sitter spacetime and the Minkowski spacetime.

On the other side, the cosmic string spacetime is a typical curved spacetime with nontrivial topological defect. The topological defect of cosmic string is produced during the evolution of the early universe due to the symmetry breaking phase transitions [7-10]. The simplest cosmic string spacetime is characterized by a deficit angle around a straight and infinitely long cosmic string, which is locally flat but not globally [11]. The nontrivial spacetime topology can give rise to some interesting physical effects, for example, bremsstrahlung process [12], gravitational lensing effect $[13,14]$ and gravitational AharonovBohm effect $[15,16]$. Furthermore, for better understanding the effects of the string, some quantum processes in the Minkowski spacetime have been extended to the cosmic string spacetime, for instance, radiative properties [17-19], Landau quantization [20,21], geometric phase [11,22], the Casimir effect [23] and so on.

As far as we know, entanglement behaviors in the cosmic string spacetime are not studied. In this work, we will investigate the entanglement behaviors for two identical accelerating atoms immersing in fluctuating massless scalar field in the background of the cosmic string spacetime. We are interested in how the nontrivial spacetime topology structure modifies the atom-field interaction and affects the entanglement behaviors, and whether entanglement behaviors can be employed to detect the spacetime topology structure. A uniformly accelerated observer can feel thermal particles in Minkowski vacuum, which is called as Unruh effect [24]; naturally, we also are intriguing with the thermal effect in cosmic string spacetime. Our investigation would throw light on the properties of the cosmic string universe and the quantum field theory of curved spacetime.

In the following, we firstly introduce and calculate the system evolution equation and entanglement measure. And then we make some derivations about the field correlation 
function for different cases, and examine the entanglement dynamics in the presence of a cosmic string. Finally, we give some conclusions of this paper.

\section{Entanglement measure for atoms in the framework of open quantum system}

The Hamiltonian of two identical atoms interacting with the massless scalar field is given by [25]

$H=H_{S}+H_{F}+H_{I}$.

The Hamiltonian $H_{S}$ of the two-level atom reads

$H_{S}=\frac{\omega}{2} \sigma_{3}^{(1)}+\frac{\omega}{2} \sigma_{3}^{(2)}$,

where $\sigma_{i}(i=1,2,3)$ are the Pauli operators and $\omega$ is the energy-level spacing between the excited state and the ground state of the atom. $H_{F}$ is the Hamiltonian of the scalar field. The interaction Hamiltonian $H_{I}$ between atoms and the scalar field is given by [25]

$H_{I}=\mu\left[\sigma_{2}^{(1)} \phi\left(t, \mathbf{x}_{1}\right)+\sigma_{2}^{(2)} \phi\left(t, \mathbf{x}_{2}\right)\right]$,

where $\mu$ is the interaction strength, $\mathbf{x}_{1}$ and $\mathbf{x}_{2}$ denotes the coordinates of the two atoms. The model is of great importance both theoretically and experimental [26-28]; by suitable adaptations, it can be applied to other different physical scenes, such as ions in traps, atoms in optical cavities and impurities in phonon fields.

Supposing that initially there is not correlation between the two atoms and the scalar field, the initial state of the total system can be written as $\rho_{\text {tot }}(0)=\rho(0) \otimes|0\rangle\langle 0|$, where $\rho(0)$ is the initial density matrix of the atoms and $|0\rangle$ is the vacuum state of the scalar field. In the weak atom-field coupling approximation, the evolution equation of the atoms is given in the Kossakowski-Lindblad form by [29-31]

$\frac{\partial \rho(\tau)}{\partial \tau}=-\mathrm{i}\left[\mathrm{H}_{\mathrm{eff}}, \rho(\tau)\right]+\mathcal{L}[\rho(\tau)]$,

where

$H_{\text {eff }}=H_{S}-\frac{i}{2} \sum_{\alpha, \beta=1}^{2} \sum_{i, j=1}^{3} H_{i j}^{(\alpha \beta)} \sigma_{i}^{(\alpha)} \sigma_{j}^{(\beta)}$,

and

$$
\begin{aligned}
\mathcal{L}[\rho]= & \frac{1}{2} \sum_{\alpha, \beta=1}^{2} \sum_{i, j=1}^{3} C_{i j}^{(\alpha \beta)}\left[2 \sigma_{j}^{(\beta)} \rho \sigma_{i}^{(\alpha)}\right. \\
& \left.-\sigma_{i}^{(\alpha)} \sigma_{j}^{(\beta)} \rho-\rho \sigma_{i}^{(\alpha)} \sigma_{j}^{(\beta)}\right] .
\end{aligned}
$$

$C_{i j}^{(\alpha \beta)}$ and $H_{i j}^{(\alpha \beta)}$ are determined by Fourier transforms $\mathcal{G}^{(\alpha \beta)}(\lambda)$ and Hilbert transforms $\mathcal{K}^{(\alpha \beta)}(\lambda)$ of field correlation function

$G^{(\alpha \beta)}\left(\tau-\tau^{\prime}\right)=\left\langle 0\left|\phi(t, \mathbf{x}) \phi\left(t^{\prime}, \mathbf{x}^{\prime}\right)\right| 0\right\rangle$,

which are defined as

$$
\begin{aligned}
\mathcal{G}^{(\alpha \beta)}(\lambda) & =\int_{-\infty}^{\infty} d \Delta \tau e^{i \lambda \Delta \tau} G^{(\alpha \beta)}, \\
\mathcal{K}^{(\alpha \Delta \tau) \beta)}(\lambda) & =\frac{P}{\pi i} \int_{-\infty}^{\infty} d \omega \frac{\mathcal{G}^{(\alpha \beta)}(\omega)}{\omega-\lambda},
\end{aligned}
$$

with $\Delta \tau=\tau-\tau^{\prime}$. The Kossakowski matrix $C_{i j}^{(\alpha \beta)}$ can be written explicitly as

$C_{i j}^{(\alpha \beta)}=A^{(\alpha \beta)} \delta_{i j}-i B^{(\alpha \beta)} \epsilon_{i j k} \delta_{3 k}-A^{(\alpha \beta)} \delta_{3 i} \delta_{3 j}$,

where

$$
\begin{aligned}
& A^{(\alpha \beta)}=\frac{\mu^{2}}{4}\left[\mathcal{G}^{(\alpha \beta)}(\omega)+\mathcal{G}^{(\alpha \beta)}(-\omega)\right], \\
& B^{(\alpha \beta)}=\frac{\mu^{2}}{4}\left[\mathcal{G}^{(\alpha \beta)}(\omega)-\mathcal{G}^{(\alpha \beta)}(-\omega)\right] .
\end{aligned}
$$

$H_{i j}^{(\alpha \beta)}$ can be obtained by replacing $\mathcal{G}^{(\alpha \beta)}$ with $\mathcal{K}^{(\alpha \beta)}$ in the above equations. Actually, Eq. (10) can be explicitly written as

$C_{i j}^{(11)}=A_{1} \delta_{i j}-i B_{1} \epsilon_{i j k} \delta_{3 k}-A_{1} \delta_{3 i} \delta_{3 j}$,

$C_{i j}^{(22)}=A_{2} \delta_{i j}-i B_{2} \epsilon_{i j k} \delta_{3 k}-A_{2} \delta_{3 i} \delta_{3 j}$,

$C_{i j}^{(12)}=C_{i j}^{(21)}=A_{3} \delta_{i j}-i B_{3} \epsilon_{i j k} \delta_{3 k}-A_{3} \delta_{3 i} \delta_{3 j}$.

Note that the natural units $c=\hbar=1$ are adopted in this article.

Supposing that the two atoms initially are prepared in the Werner state $p|\phi\rangle\langle\phi|+(1-p) \frac{I}{4}, p \in[0,1]$, with $|\phi\rangle=$ $\frac{1}{\sqrt{2}}(|00\rangle+|11\rangle)$. Werner state is separable when $p \leq \frac{1}{3}$. In this paper, we take $p=\frac{2}{3}$. Werner state [32] is an important kind of X-type that is written as

$\rho_{X}=\frac{1}{4}\left(I \otimes I+\sum_{i}^{3} c_{i} \sigma_{i} \otimes \sigma_{i}+c_{4} I \otimes \sigma_{3}+c_{5} \sigma_{3} \otimes I\right)$,

where $-1 \leq c_{i} \leq 1$. For the $\mathrm{X}$-type state, entanglement can be measured by concurrence [33], which is given by

$E=\max \left(0, F_{1}, F_{2}\right)$,

with $F_{1}=\frac{1}{2}\left[\left|c_{1}+c_{2}\right|-\sqrt{\left(c_{3}-c_{4}-c_{5}+1\right)\left(c_{3}+c_{4}+c_{5}+1\right)}\right]$ and $F_{2}=\frac{1}{2}\left[\left|c_{1}-c_{2}\right|-\sqrt{\left(c_{3}+c_{4}-c_{5}-1\right)\left(c_{3}-c_{4}+c_{5}-1\right)}\right]$. 
Arbitrary two-qubit states can use Pauli matrice to be represented as

$\rho=\frac{1}{4} \sum_{i=0}^{3} \sum_{j=0}^{3} b_{i, j}(t) \sigma_{i} \otimes \sigma_{j}$

where $\sigma_{0}$ denotes the identity matrix. As mentioned above, we considering the initial state of two atoms is Werner state. According to the initial condition, in the interaction presentation, combining Eq. (15) with Eq. (4), we only get the following non-trivial coupled differential equations

$$
\begin{aligned}
b_{0,0}^{\prime}(t)= & 0 \\
b_{1,1}^{\prime}(t)= & 2\left(-A_{1}-A_{2}\right) b_{1,1}(t)+4 A_{3} b_{3,3}(t) \\
& +2 B_{3}\left(b_{0,3}(t)+b_{3,0}(t)\right) \\
b_{0,3}^{\prime}(t)= & -4 A_{2} b_{0,3}(t)-4 B_{2} b_{0,0}(t) \\
& -2 B_{3} b_{1,1}(t)-2 B_{3} b_{2,2}(t) \\
b_{2,2}^{\prime}(t)= & 2\left(-A_{1}-A_{2}\right) b_{2,2}(t)+4 A_{3} b_{3,3}(t) \\
& +2 B_{3}\left(b_{0,3}(t)+b_{3,0}(t)\right) \\
b_{3,0}^{\prime}(t)= & -4 A_{1} b_{3,0}(t)-4 B_{1} b_{0,0}(t) \\
& -2 B_{3} b_{1,1}(t)-2 B_{3} b_{2,2}(t) \\
b_{3,3}^{\prime}(t)= & 4 A_{3} b_{1,1}(t)+4 A_{3} b_{2,2}(t)-4\left(A_{1}+A_{2}\right) b_{3,3}(t) \\
& -4\left(B_{1} b_{0,3}(t)+B_{2} b_{3,0}(t)\right) .
\end{aligned}
$$

This indicates that the two-atom evolution state is still Xtype state. According to Eq. (14), the concurrence can be expressed as

$$
\begin{aligned}
E= & \max \left[0, \frac{1}{2}\left|b_{1,1}(t)+b_{2,2}(t)\right|\right. \\
& \left.-F_{1}, \frac{1}{2}\left|b_{1,1}(t)-b_{2,2}(t)\right|-F_{2}\right],
\end{aligned}
$$

where $F_{1}=\frac{1}{2}$

$$
\begin{aligned}
& \sqrt{\left(1+b_{0,3}(t)-b_{3,0}(t)-b_{3,3}(t)\right)\left(1-b_{0,3}(t)+b_{3,0}(t)-b_{3,3}(t)\right)} \\
& \text { and } F_{2}=\frac{1}{2} \\
& \sqrt{\left(1-b_{0,3}(t)-b_{3,0}(t)+b_{3,3}(t)\right)\left(1+b_{0,3}(t)+b_{3,0}(t)+b_{3,3}(t)\right)} .
\end{aligned}
$$

\section{Quantum entanglement under cosmic string spacetime}

In this section, we discuss the entanglement dynamics of two accelerating atom immersed in a bath of fluctuating massless scalar in the background of the cosmic string spacetime. The topological defect like a cosmic string can be analogous to a disclination in crystals $[34,35]$, so our model might be simulated as moving atoms in a crystal affected by a external quantum field.
Assuming that the string lies to the $z$ axis, the spacetime line element in the cylindrical coordinates $(t, r, \theta, z)$ can be written as

$d s^{2}=d t^{2}-d r^{2}-r^{2} d \theta^{2}-d z^{2}$

where $0 \leq \theta<2 \pi / \nu, v=(1-4 G m)^{-1}$ with $G$ and $m$ being the Newton constant and the linear mass density of the string respectively. This metric is derived from the Einstein equations. The value of $m$ is related to the spontaneous symmetry breaking scale. The line element describes a locally flat spacetime with a deficit angle $8 \pi \mathrm{Gm}$.

The Klein-Gordon equation representing the massless scalar field has the form

$\partial_{t}^{2}-\frac{1}{r} \partial_{r}\left(r \partial_{r}\right)-\frac{1}{r^{2}} \partial_{\theta}^{2}-\partial_{z}^{2} \phi(t, \mathbf{x})=0$.

Solving this equation by the method of separation of variables, one can obtain a complete set of normalized field modes:

$u_{n, \kappa, k_{\perp}}(t, \mathbf{x})=\frac{1}{2 \pi} \sqrt{\frac{v}{2 \omega}} e^{-i \omega t} e^{i \kappa z} e^{i v n \theta} J_{\nu|n|}\left(k_{\perp} r\right)$,

with $\kappa \in(-\infty, \infty), n \in Z, k_{\perp} \in[0, \infty), \omega^{2}=\kappa^{2}+k_{\perp}^{2}$ and $J_{\nu|n|}\left(k_{\perp} r\right)$ being the first kind Bessel function. According to the field modes, the field operator can be expressed as

$\phi(t, \mathbf{x})=\int d \mu_{j}\left[b_{j} u_{j}(t, \mathbf{x})+\right.$ H.c. $]$

with $j \equiv\left\{n, \kappa, k_{\perp}\right\}$ and

$\int d \mu_{j}=\sum_{n=-\infty}^{\infty} \int_{-\infty}^{\infty} d \kappa \int_{0}^{\infty} d k_{\perp} k_{\perp}$.

Then the field correlation function in the cosmic string spacetime can be written as

$$
\begin{aligned}
\left\langle 0\left|\phi(t, \mathbf{x}) \phi\left(t^{\prime}, \mathbf{x}^{\prime}\right)\right| 0\right\rangle & \\
= & \frac{v}{8 \pi^{2}} \sum_{n=-\infty}^{\infty} \int_{-\infty}^{\infty} d \kappa \int_{0}^{\infty} d k_{\perp} \frac{k_{\perp}}{\omega} e^{-i \omega \Delta t} e^{i \kappa \Delta z} \\
& \times e^{i \nu n \Delta \theta} J_{v|n|}\left(k_{\perp} r\right) J_{v|n|}\left(k_{\perp} r^{\prime}\right)
\end{aligned}
$$

where $\omega=\sqrt{\kappa^{2}+k_{\perp}^{2}}, \Delta t=t-t^{\prime}, \Delta z=z-z^{\prime}$, and $\Delta \theta=$ $\theta-\theta^{\prime}$. After some transformations and by use of integral table $[18,36]$, the correlation function can be obtained

$$
\begin{aligned}
& \left\langle 0\left|\phi(t, \mathbf{x}) \phi\left(t^{\prime}, \mathbf{x}^{\prime}\right)\right| 0\right\rangle \\
& =\frac{v}{4 \pi^{2} R_{1} R_{2}} \frac{1-\Lambda^{2 v}}{1+\Lambda^{2 v}-2 \Lambda^{v} \cos (v \Delta \theta)},
\end{aligned}
$$


where

$R_{1}=\sqrt{\left(r-r^{\prime}\right)^{2}+\left(z-z^{\prime}\right)^{2}-\left(t-t^{\prime}-i \varepsilon\right)^{2}}$,

$R_{2}=\sqrt{\left(r+r^{\prime}\right)^{2}+\left(z-z^{\prime}\right)^{2}-\left(t-t^{\prime}-i \varepsilon\right)^{2}}$,

$\Lambda=\frac{R_{2}-R_{1}}{R_{2}+R_{1}}$.

The trajectories of two accelerating atoms (vertical to the string) moving along the $z$-direction can be described as

$t_{1}(\tau)=\frac{1}{a} \sinh a \tau, \quad z_{1}(\tau)=\frac{1}{a} \cosh a \tau$,

$r_{1}(\tau)=r, \quad \theta_{1}(\tau)=0$,

$t_{2}(\tau)=\frac{1}{a} \sinh a \tau, \quad z_{2}(\tau)=\frac{1}{a} \cosh a \tau$,

$r_{2}(\tau)=r+L, \quad \theta_{2}(\tau)=0$.

If $v$ is a noninteger, the calculation about correlation function is complicated, while for some special cases of integer $v$, the Fourier transform of correlation function can be derived by utilizing contour integration.

Let us firstly assume $v=1$. By Eq. (25), we obtain

$\left\langle 0\left|\phi(t, \mathbf{x}) \phi\left(t^{\prime}, \mathbf{x}^{\prime}\right)\right| 0\right\rangle=\frac{1}{4 \pi^{2} R_{1}^{2}}$.

Substituting the trajectories (27) into Eq. (28), one can obtain the correlation function

$$
\begin{aligned}
G^{(11)}\left(\tau-\tau^{\prime}\right) & =G^{(22)}\left(\tau-\tau^{\prime}\right) \\
& =-\frac{1}{16 \pi^{2}} \frac{a^{2}}{\sinh ^{2}\left(\frac{a \Delta \tau}{2}-i \epsilon\right)}, \\
G^{(12)}\left(\tau-\tau^{\prime}\right) & =G^{(21)}\left(\tau-\tau^{\prime}\right) \\
& =-\frac{1}{16 \pi^{2}} \frac{a^{2}}{\sinh ^{2}\left(\frac{a \Delta \tau}{2}-i \epsilon\right)-a^{2} L^{2} / 4} .
\end{aligned}
$$

Then Fourier transforms of the correlation function are:

$$
\begin{aligned}
& \mathcal{G}^{(11)}(\lambda)=\mathcal{G}^{(22)}(\lambda)=\frac{1}{2 \pi} \frac{\lambda}{1-e^{-2 \pi \lambda / a}}, \\
& \mathcal{G}^{(12)}(\lambda)=\mathcal{G}^{(21)}(\lambda)=\frac{1}{2 \pi} \frac{\lambda}{1-e^{-2 \pi \lambda / a}} f(\lambda, L / 2) .
\end{aligned}
$$

where $f(\lambda, s)=\frac{\sin \left[\frac{2 \lambda}{a} \sinh ^{-1}(a s)\right]}{2 s \sqrt{1+a^{2} s^{2}} \lambda}$. In terms of Eq. (11), we get

$$
A_{1}=A_{2}=\frac{\Gamma \operatorname{coth}(\pi \omega / a)}{4}, \quad B_{1}=B_{2}=\frac{\Gamma}{4},
$$

$$
A_{3}=\frac{\Gamma \operatorname{coth}(\pi \omega / a)}{4} f(\omega, L / 2), \quad B_{3}=\frac{\Gamma}{4} f(\omega, L / 2),
$$

where $\Gamma=\mu^{2} \omega / 2 \pi$ is the spontaneous emission rate of the atoms. From above equations, we know that the results reduce to the results in Minkowski spacetime [3,37].

When $v=2$, the correlation function (25) turns to

$G\left(\tau-\tau^{\prime}\right)=\frac{1}{4 \pi^{2}}\left(\frac{1}{R_{1}^{2}}+\frac{1}{R_{2}^{2}}\right)$

Combining the trajectories (27) with Eq. (32), one can obtain

$$
\begin{aligned}
& G^{(11)}\left(\tau-\tau^{\prime}\right)=-\frac{1}{16 \pi^{2}} \frac{a^{2}}{\sinh ^{2}\left(\frac{a \Delta \tau}{2}-i \epsilon\right)} \\
& -\frac{1}{16 \pi^{2}} \frac{a^{2}}{\sinh ^{2}\left(\frac{a \Delta \tau}{2}-i \epsilon\right)-a^{2} r^{2}}, \\
& G^{(22)}\left(\tau-\tau^{\prime}\right)=-\frac{1}{16 \pi^{2}} \frac{a^{2}}{\sinh ^{2}\left(\frac{a \Delta \tau}{2}-i \epsilon\right)}- \\
& \quad \frac{1}{16 \pi^{2}} \frac{a^{2}}{\sinh ^{2}\left(\frac{a \Delta \tau}{2}-i \epsilon\right)-a^{2}(r+L)^{2}}, \\
& =-\frac{1}{16 \pi^{2}} \frac{\sinh ^{2}\left(\frac{a \Delta \tau}{2}-i \epsilon\right)-a^{2} L^{2} / 4}{\left.\sin ^{(12)}\left(\tau-\tau^{\prime}\right)=G^{\prime}\right)} \\
& -\frac{1}{16 \pi^{2}} \frac{a^{2}}{\sinh ^{2}\left(\frac{a \Delta \tau}{2}-i \epsilon\right)-a^{2}(L+2 r)^{2} / 4} .
\end{aligned}
$$

The corresponding Fourier transforms are

$$
\begin{aligned}
\mathcal{G}^{(11)}(\lambda) & =\frac{1}{2 \pi} \frac{\lambda}{1-e^{-2 \pi \lambda / a}}[1+f(\lambda, r)], \\
\mathcal{G}^{(22)}(\lambda) & =\frac{1}{2 \pi} \frac{\lambda}{1-e^{-2 \pi \lambda / a}}[1+f(\lambda, r+L)], \\
\mathcal{G}^{(12)}(\lambda) & =\mathcal{G}^{(21)}(\lambda) \\
& =\frac{1}{2 \pi} \frac{\lambda}{1-e^{-2 \pi \lambda / a}}[f(\lambda, L / 2)+f(\lambda, L / 2+r)] .
\end{aligned}
$$

By Eq. (11), we obtained

$$
\begin{aligned}
& A_{1}=\frac{\Gamma \operatorname{coth}(\pi \omega / a)}{4}[1+f(\omega, r)], \\
& B_{1}=\frac{\Gamma}{4}[1+f(\omega, r)], \\
& A_{2}=\frac{\Gamma \operatorname{coth}(\pi \omega / a)}{4}[1+f(\omega, r+L)], \\
& B_{2}=\frac{\Gamma}{4}[1+f(\omega, r+L)], \\
& A_{3}=\frac{\Gamma \operatorname{coth}(\pi \omega / a)}{4}[f(\omega, L / 2)+f(\omega, L / 2+r)],
\end{aligned}
$$




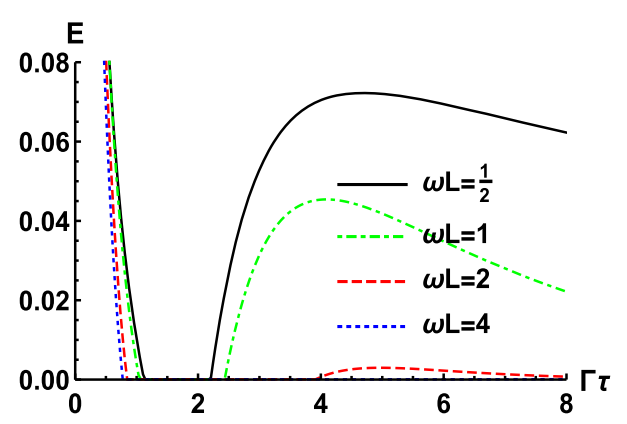

Fig. 1 Entanglement as function of $\Gamma \tau$ with different atomic separations for initial Werner state with $\mathrm{p}=2 / 3, v=2, a / \omega=1 / 2$ and $\omega r=1$

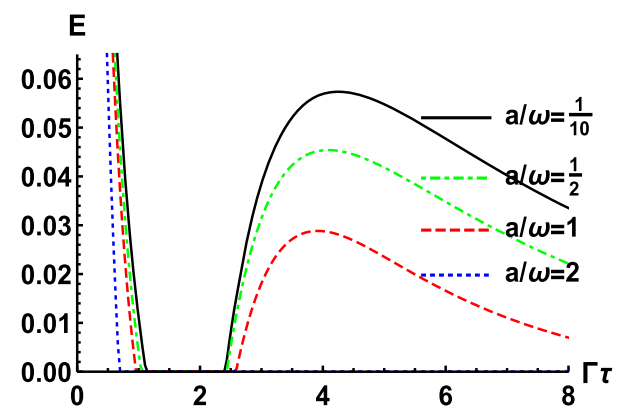

Fig. 2 Entanglement as function of $\Gamma \tau$ with different accelerations for initial Werner state with $\mathrm{p}=2 / 3, v=2, \omega L=1$ and $\omega r=1$

$B_{3}=\frac{\Gamma}{4}[f(\omega, L / 2)+f(\omega, L / 2+r)]$.

When an atom is very close to the string $(r \rightarrow 0)$, we get

$$
\begin{aligned}
& A_{1}=\frac{\Gamma \operatorname{coth}(\pi \omega / a)}{2}, \quad B_{1}=\frac{\Gamma}{2}, \\
& A_{2}=\frac{\Gamma \operatorname{coth}(\pi \omega / a)}{4}[1+f(\omega, L)], \\
& B_{2}=\frac{\Gamma}{4}[1+f(\omega, L)], \\
& A_{3}=\frac{\Gamma \operatorname{coth}(\pi \omega / a)}{2} f(\omega, L / 2), \quad B_{3}=\frac{\Gamma}{2} f(\omega, L / 2) .
\end{aligned}
$$

From Fig. 1, it can be seen that entanglement presents a gap of time during which entanglement vanishes. When twoatom distance becomes larger, entanglement decays more faster, the dark period of entanglement becomes longer, and entanglement lifetime becomes shorter; in particular, when two-atom distance is relatively big, entanglement revival no longer occurs. In this sense, we can say small two-atom distance can protect entanglement.

From Fig. 2, it can be seen that when acceleration becomes greater, entanglement decreases more, entanglement stay longer during dark period, generated entanglement keeps shorter time; in particular, when acceleration is relatively great, entanglement sudden birth no longer appears.
From Fig. 3a, it can be observed that when atom-string distance is relatively small, entanglement firstly declines and then rises with decreasing atom-string distance; specially, when atom is very close to the string, entanglement can last longer time. When acceleration is small and atom-string distance is relatively large, entanglement presents some fluctuations (see Fig. 3b). When two atoms are far away from the string $(r \rightarrow \infty)$, we exactly obtain the Eq. (31), which indicates the string has no effect on the atoms, and reduces to the case of Minkowski spacetime. Thus the atom-string distance can considerably affects the field vacuum fluctuating and atom-field interplay, and therefore adjusting the atomstring distance could be useful to detect the cosmic string. The different entanglement behaviors can in principle distinguish the cosmic string spacetime and Minkowski spacetime. When $v=3$, the correlation function (25) can be written as

$G\left(\tau-\tau^{\prime}\right)=\frac{1}{4 \pi^{2}}\left(\frac{1}{R_{1}^{2}}+\frac{8}{R_{1}^{2}+3 R_{2}^{2}}\right)$.

With similar calculating method and process, we obtained

$$
\begin{aligned}
A_{1}= & \frac{\Gamma \operatorname{coth}(\pi \omega / a)}{4}\left[1+2 f\left(\lambda, \frac{\sqrt{3} r}{2}\right)\right], \\
B_{1}= & \frac{\Gamma}{4}\left[1+2 f\left(\lambda, \frac{\sqrt{3} r}{2}\right)\right], \\
A_{2}= & \frac{\Gamma \operatorname{coth}(\pi \omega / a)}{4}\left[1+2 f\left(\lambda, \frac{\sqrt{3} r+\sqrt{3} L}{2}\right)\right], \\
B_{2}= & \frac{\Gamma}{4}\left[1+2 f\left(\lambda, \frac{\sqrt{3} r+\sqrt{3} L}{2}\right)\right], \\
A_{3}= & \frac{\Gamma \operatorname{coth}(\pi \omega / a)}{4}[f(\omega, L / 2) \\
& \left.+2 f\left(\lambda, \sqrt{L^{2}+3 L r+3 r^{2}} / 2\right)\right], \\
B_{3}= & \frac{\Gamma}{4}\left[f(\omega, L / 2)+2 f\left(\lambda, \sqrt{L^{2}+3 L r+3 r^{2}} / 2\right)\right] .
\end{aligned}
$$

When an atom is very close to the string $(r \rightarrow 0)$, we get

$$
\begin{aligned}
& A_{1}=\frac{3 \Gamma \operatorname{coth}(\pi \omega / a)}{4}, \quad B_{1}=\frac{3 \Gamma}{4}, \\
& A_{2}=\frac{\Gamma \operatorname{coth}(\pi \omega / a)}{4}\left[1+2 f\left(\lambda, \frac{\sqrt{3} L}{2}\right)\right], \\
& B_{2}=\frac{\Gamma}{4}\left[1+f\left(\omega, \frac{\sqrt{3} L}{2}\right)\right], \\
& A_{3}=\frac{3 \Gamma \operatorname{coth}(\pi \omega / a)}{4} f(\omega, L / 2), \\
& B_{3}=\frac{3 \Gamma}{4} f(\omega, L / 2) .
\end{aligned}
$$


Fig. 3 Entanglement as function of $\Gamma \tau$ with different Werner state with $\mathrm{p}=2 / 3$, $v=2, a / \omega=1 / 2$ and $\omega L=1 / 2$

Fig. 4 Entanglement as function of $\Gamma \tau$ with different atom-string distances for initial Werner state with $\mathrm{p}=2 / 3, v=3$, $a / \omega=1 / 2$ and $\omega L=1 / 2$ atom-string distances for initial

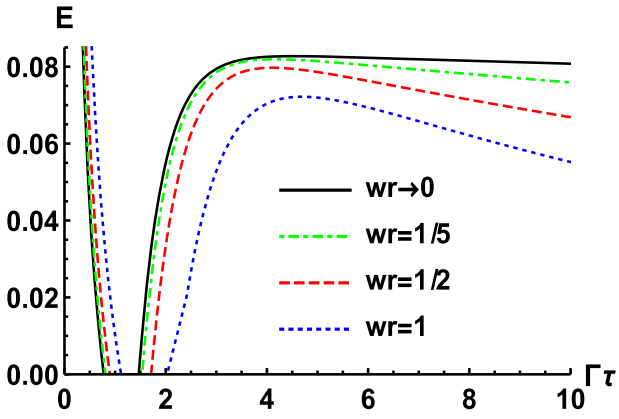

(a)

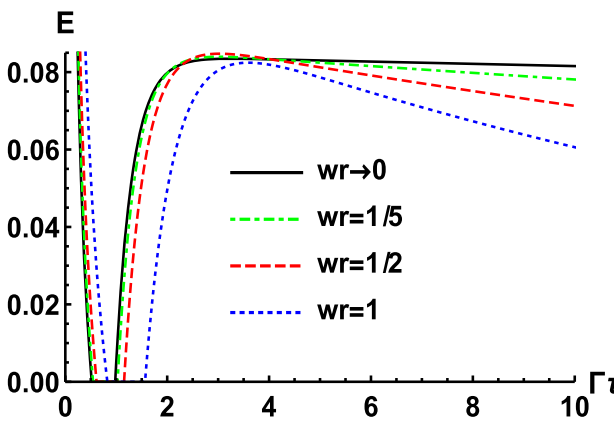

(a)

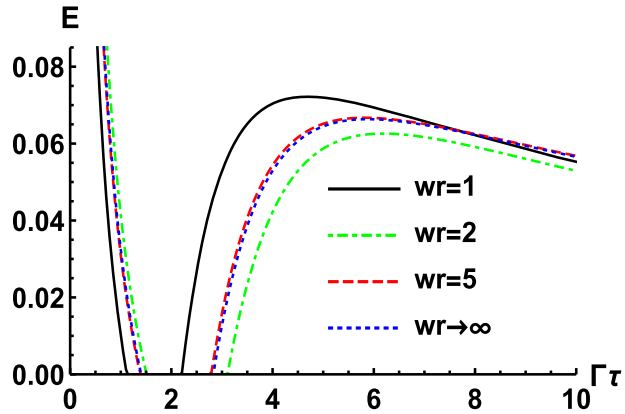

(b)

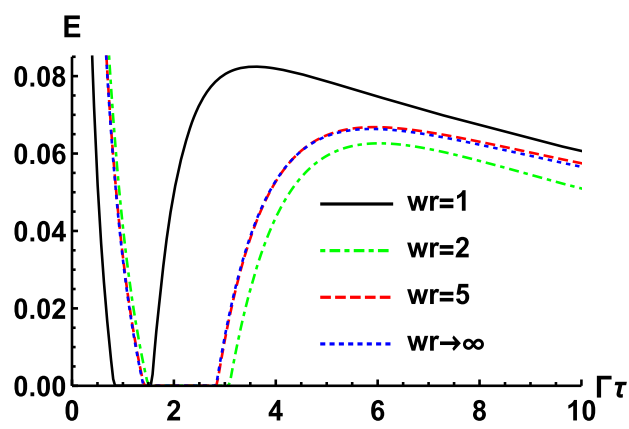

(b)
When atoms are far away from the string $(r \rightarrow \infty)$, the Eq. (31) is be obtained. Entanglement variations displayed in Fig. 4 are similar with Fig. 3. But entanglement presents more obvious fluctuations.

Finally, we investigate the Unruh thermal effect in the cosmic string spacetime. by letting the rates of change of the coefficients in Eq. (17) to be zero, we can solve the relevant

$$
\begin{aligned}
\rho_{\infty} & =\frac{1}{4}\left(\begin{array}{cccc}
\left(\tanh \frac{\pi \omega}{a}-1\right)^{2} & 0 & 0 & 0 \\
0 & \operatorname{sech}^{2} \frac{\pi \omega}{a} & 0 & 0 \\
0 & 0 & \operatorname{sech}^{2} \frac{\pi \omega}{a} & 0 \\
0 & 0 & 0 & \left(\tanh \frac{\pi \omega}{a}+1\right)^{2}
\end{array}\right) \\
& =\frac{e^{-H_{S} / T}}{\operatorname{Tr}\left(e^{-H_{S} / T}\right)},
\end{aligned}
$$

$$
\begin{aligned}
& b_{0,3}(\tau)=\frac{\left(A_{1}+A_{2}\right)\left(B_{1}-B_{2}\right) B_{3}^{2}+2 A_{3} B_{2}\left(B_{2}-B_{1}\right) B_{3}-A_{1}\left[3\left(A_{1}+A_{2}\right)+2 A_{3}\right]\left(A_{1}+A_{2}-2 A_{3}\right) B_{2}}{\left(A_{1}+A_{2}\right)^{2} B_{3}^{2}-2 A_{3} B_{3}\left(A_{1} B_{1}+A_{2} B_{2}\right)+A_{1} A_{2}\left(3\left(A_{1}+A_{2}\right)+2 A_{3}\right)\left(A_{1}+A_{2}-2 A_{3}\right)}, \\
& b_{1,1}(\tau)=-\frac{\left(A_{1}+A_{2}\right)\left[B_{3}\left(A_{2} B_{1}+A_{1} B_{2}\right)-2 A_{3} B_{1} B_{2}\right]}{\left(A_{1}+A_{2}\right)^{2} B_{3}^{2}-2 A_{3} B_{3}\left(A_{1} B_{1}+A_{2} B_{2}\right)+A_{1} A_{2}\left[3\left(A_{1}+A_{2}\right)+2 A_{3}\right]\left(A_{1}+A_{2}-2 A_{3}\right)}, \\
& b_{2,2}(\tau)=-\frac{\left(A_{1}+A_{2}\right)\left[B_{3}\left(A_{2} B_{1}+A_{1} B_{2}\right)-2 A_{3} B_{1} B_{2}\right]}{\left(A_{1}+A_{2}\right)^{2} B_{3}^{2}-2 A_{3} B_{3}\left(A_{1} B_{1}+A_{2} B_{2}\right)+A_{1} A_{2}\left[3\left(A_{1}+A_{2}\right)+2 A_{3}\right]\left(A_{1}+A_{2}-2 A_{3}\right)}, \\
& b_{3,0}(\tau)=-\frac{\left(A_{1}+A_{2}\right)\left(B_{1}-B_{2}\right) B_{3}^{2}+2 A_{3} B_{1}\left(B_{2}-B_{1}\right) B_{3}+A_{2}\left[3\left(A_{1}+A_{2}\right)+2 A_{3}\right]\left(A_{1}+A_{2}-2 A_{3}\right) B_{1}}{\left(A_{1}+A_{2}\right)^{2} B_{3}^{2}-2 A_{3} B_{3}\left(A_{1} B_{1}+A_{2} B_{2}\right)+A_{1} A_{2}\left[3\left(A_{1}+A_{2}\right)+2 A_{3}\right]\left(A_{1}+A_{2}-2 A_{3}\right)}, \\
& b_{3,3}(\tau)=\frac{-2 A_{3} B_{3}\left(A_{2} B_{1}+A_{1} B_{2}\right)+\left(A_{1}+A_{2}\right)\left[3\left(A_{1}+A_{2}\right)-4 A_{3}\right] B_{1} B_{2}-\left(B_{1}-B_{2}\right)^{2} B_{3}^{2}}{\left(A_{1}+A_{2}\right)^{2} B_{3}^{2}-2 A_{3} B_{3}\left(A_{1} B_{1}+A_{2} B_{2}\right)+A_{1} A_{2}\left[3\left(A_{1}+A_{2}\right)+2 A_{3}\right]\left(A_{1}+A_{2}-2 A_{3}\right)} .
\end{aligned}
$$

Substituting Eq. (31), Eq. (35) or Eq. (38) into Eq. (40), we can obtain the equilibrium state where $T=\frac{a}{2 \pi}$ denotes the Unruh temperature. The fact that Eq. (41) is a separable state indicates that entanglement can not persist over time. Equation (41) reveals that the twoatom system evolves to a thermal state, and manifests the Unruh thermal effect in the cosmic string spacetime. This demonstrates cosmic string spacetime is locally flat, unlike 
the de Sitter spacetime shows the Gibbons-Hawking thermal effect of curved spacetime [38].

\section{Conclusion}

We have investigated the entanglement dynamics for two accelerating atoms immersing in a bath of fluctuating massless scalar field in the cosmic string spacetime. We concretely calculate the entanglement related quantities of different cases for two accelerating atoms vertical to the infinite and straight cosmic string. It is shown that vacuum fluctuation, two-atom separation, acceleration, atom-string distance and deficit angle parameter affect the entanglement behaviors. For deficit angle parameter $v=1$, the cosmic string spacetime reduces to the Minkowski spacetime. Small twoatom separation could make entanglement gain better protection. Great acceleration can quicken entanglement degradation. When atom is near to the string, entanglement behaviors become complicated, which indicates the string can make profoundly modifications to the atom-field interaction and vacuum fluctuation. In general, when atom is relatively close to the string, entanglement decays more slowly and has longer lifetime. The two-atom distance, acceleration, deficit angle parameter and atom-string distance give us more freedom to adjust the entanglement behaviors, which in principle is helpful to detect the different cosmic string spacetimes, and distinguish the cosmic string universe from the Minkowski universe.

Data Availability Statement This manuscript has no associated data or the data will not be deposited. [Authors' comment: There are no other data associated with the manuscript.]

Open Access This article is licensed under a Creative Commons Attribution 4.0 International License, which permits use, sharing, adaptation, distribution and reproduction in any medium or format, as long as you give appropriate credit to the original author(s) and the source, provide a link to the Creative Commons licence, and indicate if changes were made. The images or other third party material in this article are included in the article's Creative Commons licence, unless indicated otherwise in a credit line to the material. If material is not included in the article's Creative Commons licence and your intended use is not permitted by statutory regulation or exceeds the permitted use, you will need to obtain permission directly from the copyright holder. To view a copy of this licence, visit http://creativecomm ons.org/licenses/by/4.0/.

Funded by SCOAP ${ }^{3}$.

\section{References}

1. M.A. Nilsen, I.L. Chuang, Quantum Computation and Quantum Information (Cambridge University Press, Cambridge, 2000)

2. R. Horodecki, P. Horodecki, M. Horodecki, K. Horodecki, Quantum entanglement. Rev. Mod. Phys. 81, 865 (2009)
3. J.W. Hu, H.W. Yu, Entanglement dynamics for uniformly accelerated two-level atoms. Phys. Rev. A 91, 012327 (2015)

4. Y.Q. Yang, J.W. Hu, H.W. Yu, Entanglement dynamics for uniformly accelerated two-level atoms coupled with electromagnetic vacuum fluctuations. Phys. Rev. A 94, 032337 (2016)

5. J.W. Hu, H.W. Yu, Quantum entanglement generation in de Sitter spacetime. Phys. Rev. D 88, 104003 (2013)

6. Z.M. Huang, Z.H. Tian, Dynamics of quantum entanglement in de Sitter spacetime and thermal Minkowski spacetime. Nucl. Phys. B 923, 458 (2017)

7. A. Velenkin, E.P.S. Shellard, Cosmic Strings and Other Topological Defects (Cambridge University Press, Cambridge, 1994)

8. E.J. Copeland, L. Pogosian, T. Vachaspati, Seeking string theory in the cosmos. Class. Quantum Gravity 28, 204009 (2011)

9. M. Hindmarsh, Signals of inflationary models with cosmic strings. Prog. Theor. Phys. Suppl. 190, 197 (2011)

10. H.F.S. Mota, M. Hindmarsh, Big-bang nucleosynthesis and gamma-ray constraints on cosmic strings with a large Higgs condensate. Phys. Rev. D 91, 043001 (2015)

11. H. Cai, Z. Ren, Geometric phase for a static two-level atom in cosmic string spacetime. Class. Quantum Gravity 35, 105014 (2018)

12. J. Audretsch, A. Economou, Conical bremsstrahlung in a cosmicstring spacetime. Phys. Rev. D 44, 3774 (1991)

13. A. Vilenkin, Cosmic strings as gravitational lenses. Astrophys. J. 282, L51 (1984)

14. J.R. Gott, Gravitational lensing effects of vacuum strings-exact solutions. Astrophys. J. 288, 422 (1985)

15. L.H. Ford, A. Vilenkin, A gravitational analogue of the AharonovCBohm effect. J. Phys. A Math. Gen. 14, 2353 (1981)

16. V.B. Bezerra, Gravitational analogs of the Aharonov-Bohm effect. J. Math. Phys. 30, 2895 (1989)

17. H. Cai, H. Yu, W. Zhou, Spontaneous excitation of a static atom in a thermal bath in cosmic string spacetime. Phys. Rev. D 92, 084062 (2015)

18. W. Zhou, H. Yu, Spontaneous excitation of a uniformly accelerated atom in the cosmic string spacetime. Phys. Rev. D 93, 084028 (2016)

19. H. Cai, Z. Ren, Radiative processes of two entangled atoms in cosmic string spacetime. Class. Quantum Gravity 35, 025016 (2018)

20. K. Bakke, L.R. Ribeiro, C. Furtado, J.R. Nascimento, Landau quantization for a neutral particle in the presence of topological defects. Phys. Rev. D 79, 024008 (2009)

21. E.R. Figueiredo Medeiros, E.R.Bezerra de Mello, Relativistic quantum dynamics of a charged particle in cosmic string spacetime in the presence of magnetic field and scalar potential. Eur. Phys. J. C 72, 2051 (2012)

22. K. Bakke, J.R. Nascimento, C. Furtado, Geometric phase for a neutral particle in the presence of a topological defect. Phys. Rev. D 78, 064012 (2008)

23. E.R. Bezerra de Mello, A.A. Saharian, A. Kh Grigoryan, Casimir effect for parallel metallic plates in cosmic string spacetime. J. Phys. A Math. Theor. 45, 374011 (2012)

24. W.G. Unruh, Notes on black-hole evaporation. Phys. Rev. D 14, 870 (1976)

25. J. Audretsch, R. Müller, Spontaneous excitation of an accelerated atom: the contributions of vacuum fluctuations and radiation reaction. Phys. Rev. A 50, 1755 (1994)

26. P.W. Milonni, The Quantum Vacuum: An Introduction to Quantum Electrodynamics (Academic, San Diego, 1994)

27. Z. Ficek, R. Tanas, Entangled states and collective nonclassical effects in two-atom systems. Phys. Rep. 372, 369 (2002)

28. R.R. Puri, Mathematical Methods of Quantum Optics (Springer, Berlin, 2001)

29. V. Gorini, A. Kossakowski, E.C.G. Surdarshan, Completely positive dynamical semigroups of N-level systems. J. Math. Phys. 17, 821 (1976) 
30. G. Lindblad, On the generators of quantum dynamical semigroups. Commun. Math. Phys. 48, 119 (1976)

31. H.-P. Breuer, F. Petruccione, The Theory of Open Quantum Systems (Oxford University Press, Oxford, 2002)

32. R.F. Werner, Quantum states with Einstein-Podolsky-Rosen correlations admitting a hidden-variable model. Phys. Rev. A 40, 4277 (1989)

33. W.K. Wootters, Entanglement of formation of an arbitrary state of two qubits. Phys. Rev. Lett. 80, 2245 (1998)

34. F. Moraes, Condensed matter physics as a laboratory for gravitation and cosmology. Braz. J. Phys. 30, 304 (2000)

35. Y.M. Bunkov, H. Godfrin, Topological Defects and the NonEquilibrium Dynamics Of Symmetry Breaking Phase Transitions (Springer, Berlin, 2012)
36. I.S. Gradshteyn, I.M. Ryzhik, Table of Integrals, Series, and Products, 7th edn. (Academic, Orlando, 1980)

37. Z.M. Huang, Behaviors of quantum correlation for accelerated atoms coupled with a fluctuating massless scalar field with a perfectly reflecting boundary. Quantum Inf. Process. 18, 187 (2019)

38. H.W. Yu, Open quantum system approach to the Gibbons-Hawking effect of de Sitter space-time. Phys. Rev. Lett. 106, 061101 (2011) 